\title{
Differences in the Association between Clinically Relevant Classifications of Glycemia Measures and All-Cause and Cardiovascular Disease Mortality Risk
}

\author{
Jacinta I. Reddigan, Chris I. Ardern, Michael C. Riddell and Jennifer L. Kuk* \\ School of Kinesiology and Health Science, York University, Toronto, Canada
}

\begin{abstract}
Aims: To examine all-cause and cardiovascular disease (CVD) mortality risk in individuals categorized as normal, impaired or type 2 diabetic using clinical cutoffs for fasting plasma glucose (FPG), 2-hour plasma glucose (2hPG) and glycated hemoglobin (HbA1c).

Methods: The sample included 5,424 adults with undiagnosed diabetes from the Third National Health and Nutrition Examination Survey with public-access mortality data linkage (follow-up $=8.5 \pm 2.3$ years; 685 deaths). The association between the glycemic measures and all-cause and CVD mortality were analyzed with the measures as continuous and categorical variables. FPG, $2 \mathrm{hPG}$ and $\mathrm{HbA} 1 \mathrm{c}$ were categorized using the American Diabetes Association criteria for normal, impaired and type 2 diabetes.

Results: When analyzed as a continuous variable, $2 \mathrm{hPG}$ was most strongly associated with both all-cause and CVD mortality. However, after categorizing each measure using clinical cutoffs, impaired and type 2 diabetic levels of FPG and $\mathrm{HbA} 1 \mathrm{c}$, but only type 2 diabetic levels of $2 \mathrm{hPG}$, were significantly associated with all-cause mortality. For CVD mortality, impaired (HR=1.68 [1.16-2.44]) and type 2 diabetic $(H R=1.88$ [1.11-3.18)] levels of HbA1c were found to be a significant predictor of mortality risk. However, only type 2 diabetic levels of $2 \mathrm{hPG}$ and not impaired levels were significantly associated with CVD mortality. FPG was not a significant predictor of CVD mortality.

Conclusions: Clinically relevant categories of $\mathrm{HbA} 1 \mathrm{c}$ provide more prognostic information for all-cause and CVD mortality risk than $2 \mathrm{hPG}$. Therefore, given the ease and lower cost of measurement, HbA1c should be considered a beneficial diagnostic and prognostic alternative screening tool in the clinical setting.
\end{abstract}

Keywords: Epidemiology; Clinical diabetes; Diagnosis; HbA1c

Abbreviations: CDC: Centers for Disease Control and Prevention; CVD: Cardiovascular Disease; FPG: Fasting plasma glucose; HbA1c: Glycated haemoglobin; 2hPG: 2 hour plasma glucose; HR: Hazard ratio; NHANES: National Health and Nutrition Examination Survey; OGTT: Oral glucose tolerance test

\section{Introduction}

The 2-hour oral glucose tolerance test (OGTT) is the gold standard for diagnosing type 2 diabetes. However, its use in the clinical setting can be limited due to its high cost and labor-intensive multi-blood draw protocols [1]. Additionally, this measure requires patients to be tested after an overnight fast, which may be difficult for many individuals and confirmation of adherence is not possible [2]. Fasting glucose (FPG) is a cheaper single point surrogate measure, but still requires patients to be tested in the fasted state. Moreover, a significant percentage of individuals can have glucose intolerance but normal fasting glucose levels [3]. Glycated haemoglobin (HbA1c), like fasting glucose, requires only a single point blood draw, but has the added advantage in that it does not require fasting blood samples and has higher repeatability [4]. HbA1c is an indicator of the average blood glucose concentration over the preceding three months [5] and has been proposed to be a useful alternative test to screen for type 2diabetes as it overcomes many of the obstacles associated with the OGTT.

In addition to diagnostic purposes for type 2 diabetes, glucose tolerance, fasting plasma glucose (FPG) and HbA1c can also provide additional prognostic information regarding mortality risk. FPG [6,7] 2-hour plasma glucose (2hPG) [8,9] and HbA1c [10,11] have all been shown to be associated with both cardiovascular and all- cause mortality, but few studies have directly compared the ability of these three glycemic measures to predict mortality risk [12-17]. When using continuous or distributional cut-offs, the majority of studies to date report that 2hPG [13-15] or both 2hPG and FPG [12] are stronger predictors of all-cause and cardiovascular disease (CVD) mortality in comparison to $\mathrm{HbA1c}$. Despite the weighted literature, physicians still value the clinical utility of $\mathrm{HbA} 1 \mathrm{c}$ and often use this measure to determine the effectiveness of type 2 diabetes treatment in lieu of the OGTT [18]. In contrast, a recent meta-analysis [19] found that $\mathrm{HbA1c}$ was more strongly associated with coronary heart disease than 2hPG or FPG. However, this study was limited in that observations made in meta-analyses are indirect as associations are often compared between difference groups of individuals. Further, this study did not compare clinical classifications of glycemia (ie. impaired or type 2diabetes versus normal). Only one study to date [13] has directly examined the clinical utility of the three glycemic measure in predicting mortality risk, in which clinical cutoffs were used to create categories of FPG, $2 \mathrm{hPG}$ and HbA1c. This study

*Corresponding author: Jennifer L. Kuk PhD, School of Kinesiology and Health Science, York University, Toronto, Ontario, Canada, Tel: 416-736-2100 X 20289; Fax: 416-736-5774; Email: jennkuk@yorku.ca

Received October 18, 2010; Accepted October 30, 2010; Published November 01, 2010

Citation: Reddigan JI, Ardern Cl, Riddell MC, Kuk JL (2010) Differences in the Association between Clinically Relevant Classifications of Glycemia Measures and All-Cause and Cardiovascular Disease Mortality Risk. J Diabetes Metab 1:106 doi:10.4172/2155-6156.1000106

Copyright: (c) 2010 Reddigan $\mathrm{JI}$, et al. This is an open-access article distributed under the terms of the Creative Commons Attribution License, which permits unrestricted use, distribution, and reproduction in any medium, provided the original author and source are credited. 
reported that only the type 2 diabetic category of $2 \mathrm{hPG}$ and not HbA1c was significantly associated with all-cause mortality. However this study was conducted in an older population (50-75 years) with a smaller sample size $(\mathrm{N}=2,363)$, hence, the relative clinical utility of the FPG, $2 \mathrm{hPG}$ and $\mathrm{HbA1c}$ requires further investigation.

Therefore, the aim of the current study is to examine all-cause and CVD mortality risk in individuals categorized as normal, impaired or type 2 diabetic using clinical cut-offs for FPG, 2hPG and HbA1c in a representative population of U.S. adults.

\section{Patients and Methods}

The Third National Health and Nutrition Examination Survey (NHANESIII) is a nationally representative cross-sectional survey that was conducted between 1988 and 1994 by the National Center for Health Statistics of the Centers for Disease Control and Prevention, in 33,994 persons, aged 2 months or older. All study participants gave their informed written consent before participation in the examination and the study protocol was approved by the National Center for Health Statistics. The sample was collected using a multistage stratified probability cluster design. Complete details of the study design and procedures are reported elsewhere [20]. A sample of 5,424 adults (age 40-74 years) from the public access Mortality Linkage data file was used, with follow-up through December 31, 2000. Individuals were excluded if they were pregnant, were less than 18 years of age and had missing data for age, mortality follow-up, HbA1c, or $2 \mathrm{hr}$ glucose OGTT measure.

Age, sex, income $(<\$ 10,000, \$ 10,000-29,999, \$ 30,000-39,999$, $\geq \$ 40,000$ ), ethnicity (non-Hispanic White or non-White), smoking status (current smoker, past smoker, or never smoked), alcohol intake ( $\geq 3$ drinks per day or $<3$ drinks per day), being physically active (exercise frequency $\geq 5$ times per week or $<5$ times per week), dietary fat intake $(>30 \%$ or $\leq 30 \%)$ and physician diagnosed type 2 diabetes mellitus were assessed by questionnaire. Body mass index (BMI) was calculated using measured height and weight.

The OGTT was conducted at the mobile examination center in individuals aged 40-74 years old without previously diagnosed type 2 diabetes and were not taking insulin, after a complete overnight fast ( $>8$ hours). Blood was drawn from the antecubital vein by a trained phlebotomist according to a standardized protocol. Participants received a 75-g glucose-equivalent oral glucose challenge and a second blood sample was drawn 2 hours later. Fasting and 2-h plasma glucose levels were measured by a hexokinase enzymatic reference method (Roche Cobas Mira, Indianapolis, IN) [20]. HbA1c was measured by ion-exchange high-performance liquid chromatography method (Bio-Rd Diamat, Hercules, CA) from blood collected via the antecubital vein puncture [20].

FPG (<5.6, 5.6-6.9 and $\geq 7.0 \mathrm{~m} \mathrm{~mol} / \mathrm{l}), 2 \mathrm{hPG}(<7.8,7.8-11.0,>$ $11.0 \mathrm{~m} \mathrm{~mol} / \mathrm{l})$ and HBA1c $(<5.7,5.7-6.4, \geq 6.5 \%)$ were categorized to reflect the American Diabetes Association criteria for normal, impaired and diabetic concentrations [21].

\section{Statistical analysis}

FPG, 2hPG and HbA1c were analyzed as both continuous and categorical variables. Baseline characteristics were reported stratified by FPG, $2 \mathrm{hPG}$ and $\mathrm{HbA} 1 \mathrm{c}$ categories (normal, impaired, type 2 diabetes) using the clinical cut-offs. Differences in participant characteristics between categories for each glucose measurement type were assessed using either a one-way analysis of variance or a chi-square test. Unadjusted mortality rates per 1,000 person years were also calculated for each category of FPG, $2 \mathrm{hPG}$ and HbA1c. To investigate the discrepancy in diagnosis between the glycemic measures, crosstab prevalence was conducted, in addition to positive and negative predictive values.

Standardized hazard ratios (HR) for all-cause and cardiovascular mortality were estimated by Cox proportional hazard regression for FPG, 2hPG and HbA1c as continuous and categorical variables. Each model was adjusted for age, sex, income, ethnicity and smoking status, alcohol intake, being physically active and high dietary fat intake. As there were no significant sex-interactions, analyses were conducted with men and women together. Analyses were performed using SAS version 9.1 (SAS Institute, Inc., Cary, NC) or SUDDAN 10.0 (SUDAAN, Research Triangle Institute, Research Triangle Park, NC), weighted to be representative of the U.S population.

\section{Results}

During an average of $8.5 \pm 2.3$ years of follow-up there were 685 deaths, 282 of which were due to cardiovascular causes. In general, the glycemic values were in the upper normal to impaired ranges $(2 \mathrm{hPG}=7.8 \pm 0.01 \mathrm{mmol} / \mathrm{L} ; \mathrm{HbA} 1 \mathrm{c}=5.5 \pm 0.03 \%$; FPG $=5.61 \pm 0.04$ $\mathrm{mmol} / \mathrm{L})$. Men had significantly higher levels of $2 \mathrm{hPG}$, but significantly lower levels of FPG and HbA1c than women $(\mathrm{p}<0.05)$.

\begin{tabular}{|c|c|c|c|c|c|c|c|c|c|}
\hline & \multicolumn{3}{|c|}{ 2hPG (m mol/l) } & \multicolumn{3}{|c|}{ FPG (m mol/l) } & \multicolumn{3}{|l|}{ HbA1c (\%) } \\
\hline & $<7.8$ & $7.8-11.0$ & $>11.0$ & $<5.6$ & $5.6-6.9$ & $\geq 7.0$ & $<5.7$ & $5.7-6.4$ & 6.5 \\
\hline 2hPG (m mol/L) & $5.7 \pm 0.1$ & $9.2 \pm 0.1$ & $15.6 \pm 0.3$ & $7.0 \pm 0.1$ & $8.1 \pm 0.2$ & $17.8 \pm 0.5$ & $6.9 \pm 0.1$ & $8.0 \pm 0.1$ & $17.9 \pm 0.5$ \\
\hline FPG (m mol/L) & $5.3 \pm 0.1$ & $5.4 \pm 0.1$ & $7.6 \pm 0.2$ & $5.1 \pm 0.1$ & $6.0 \pm 0.1$ & $10.5 \pm 0.2$ & $5.3 \pm 0.1$ & $5.9 \pm 0.1$ & $10.0 \pm 0.3$ \\
\hline $\mathrm{HbA1c}(\%)$ & $5.3 \pm 0.1$ & $5.4 \pm 0.1$ & $6.7 \pm 0.1$ & $5.3 \pm 0.1$ & $5.5 \pm 0.1$ & $7.9 \pm 0.2$ & $5.1 \pm 0.1$ & $5.6 \pm 0.1$ & $8.2 \pm 0.1$ \\
\hline Age (yrs) & $52.8 \pm 0.3$ & $56.4 \pm 0.5^{\mathrm{a}}$ & $60.0 \pm 0.7^{a}$ & $53.7 \pm 0.3$ & $56.5 \pm 0.6^{a}$ & $57.9 \pm 0.9^{a}$ & $53.3 \pm 0.3$ & $58.0 \pm 0.4^{\mathrm{a}}$ & $58.2 \pm 0.9^{a}$ \\
\hline \multicolumn{10}{|l|}{ Ethnicity (\%) } \\
\hline White & 81.2 & 80.9 & 77.8 & 81.3 & 79.6 & $76.0^{a}$ & 84.8 & $70.0^{a}$ & $67.8^{a}$ \\
\hline Black & 9.1 & 7.9 & 8.7 & 8.7 & 8.2 & $11.6^{a}$ & 6.0 & $16.0^{a}$ & $16.5^{a}$ \\
\hline Hispanic & 3.0 & $4.01^{a}$ & $5.7^{\mathrm{a}}$ & 3.2 & $4.4^{\mathrm{a}}$ & $6.2^{a}$ & 3.0 & $4.7^{a}$ & $7.1^{\mathrm{a}}$ \\
\hline Other & 6.8 & 7.2 & 7.7 & 6.9 & 7.8 & 6.2 & 6.2 & $9.3^{\mathrm{a}}$ & 8.6 \\
\hline Body Mass Index $\left(\mathrm{kg} / \mathrm{m}^{2}\right)$ & $26.9 \pm 0.2$ & $27.9 \pm 0.2^{a}$ & $29.0 \pm 0.4^{a}$ & $26.9 \pm 0.2$ & $28.3 \pm 0.3^{a}$ & $31.0 \pm 0.5^{a}$ & $26.9 \pm 0.2$ & $28.2 \pm 0.3^{a}$ & $30.8 \pm 0.4^{a}$ \\
\hline \multicolumn{10}{|l|}{ Education Level (\%) } \\
\hline Less than high school & 23.4 & $27.6^{\mathrm{a}}$ & $37.4^{\mathrm{a}}$ & 23.7 & $31.7^{\mathrm{a}}$ & $39.1^{\mathrm{a}}$ & 22.3 & $35.0^{a}$ & $42.6^{a}$ \\
\hline High school or equivalent & 32.6 & $39.4^{\mathrm{a}}$ & 35.1 & 34.7 & 34.1 & 32.8 & 34.6 & 34.3 & 33.7 \\
\hline College or above & 44.0 & $33.1^{\mathrm{a}}$ & $27.4^{\mathrm{a}}$ & 41.6 & $34.2^{\mathrm{a}}$ & $28.1^{\mathrm{a}}$ & 43.1 & $30.8^{a}$ & $23.6^{a}$ \\
\hline
\end{tabular}

$a=p<0.05$

Plus-minus values are means \pm SD

Table 1: Characteristics of Participants in the Third National Health and Nutrition Examination Survey by categories of $2 \mathrm{hPG}, \mathrm{FPG}$ and HbA1c. 


\begin{tabular}{|c|c|c|c|c|c|c|}
\hline & \multicolumn{2}{|c|}{$2 \mathrm{hPG}(\mathrm{m} \mathrm{mol} / \mathrm{L})$} & \multicolumn{2}{|c|}{ FPG (m mol/L) } & \multicolumn{2}{|c|}{ HbA1c (\%) } \\
\hline & Impaired & T2D & Impaired & T2D & Impaired & T2D \\
\hline \multicolumn{7}{|c|}{ HbA1c (\%) } \\
\hline Normal & 21.6 & 6.9 & 18.0 & 8.0 & & \\
\hline Impaired & 29.7 & 15.2 & 32.2 & 4.6 & - & - \\
\hline T2D & 7.3 & 88.1 & 16.7 & 65.9 & - & - \\
\hline \multicolumn{7}{|l|}{ FPG (\%) } \\
\hline Normal & 21.7 & 7.5 & & & 18.0 & 1.4 \\
\hline Impaired & 28.3 & 15.9 & - & - & 32.4 & 4.9 \\
\hline T2D & 9.3 & 85.4 & - & - & 17.3 & 72.3 \\
\hline \multicolumn{7}{|l|}{ 2hPG (\%) } \\
\hline Normal & & & 18.2 & 0.46 & 18.1 & 0.43 \\
\hline Impaired & - & - & 26.3 & 2.3 & 27.8 & 2.0 \\
\hline T2D & - & - & 24.3 & 35.1 & 23.5 & 39.7 \\
\hline
\end{tabular}

FPG Normal: < 5.6, Impaired: 5.6-6.9, T2D: $\geq 7.0 \mathrm{~m} \mathrm{~mol} / \mathrm{L}$

2h-PG Normal: <7.8, Impaired: 7.8-11.0, T2D: > $11.0 \mathrm{~m} \mathrm{~mol} / \mathrm{L}$

HBA $_{1 \mathrm{c}}$ Normal: <5.7, Impaired: 5.7-6.4, T2D: $\geq 6.5 \%$

Table 2: Prevalence of concordant and discordant classifications of normal, impaired and type 2 diabetic glucose levels by three glycemic measures.

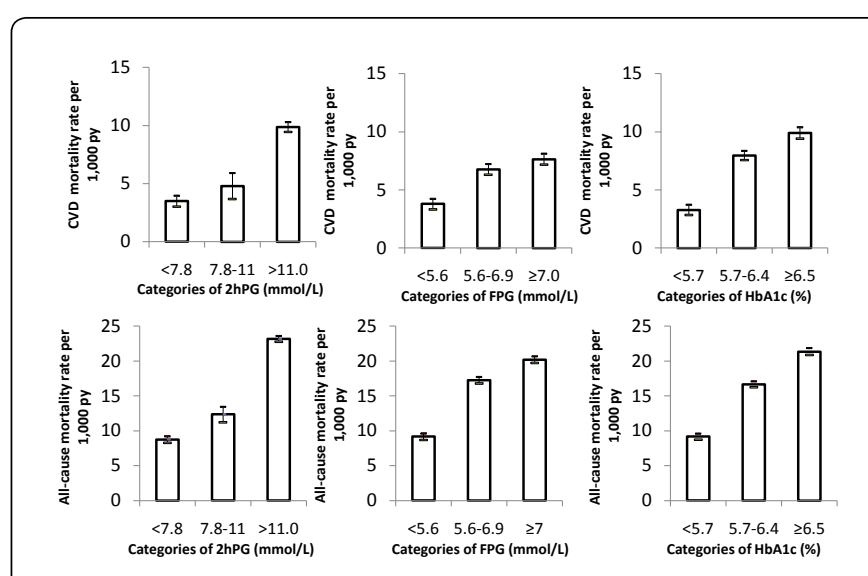

Figure 1: Unadjusted all-cause (A-C) and CVD mortality (D-F) rates per 1,000 person years $(95 \% \mathrm{Cl})$ for $2 \mathrm{hPG}, \mathrm{FPG}$ and $\mathrm{HbA} 1 \mathrm{c}$.

Characteristics of participants are shown in Table 1. In general, individuals with impaired or diabetic levels were older, more likely to be Black or Hispanic, have a higher BMI and a lower education level than those who were normoglycemic $(p<0.05)$. Figure 1 shows the crude all-cause and CVD mortality rates for each glucose measure. Both all-cause and CVD mortality rates increased from normal, impaired to type 2 diabetic concentrations of $2 \mathrm{hPG}$, FPG and HbA1c.

Table 2 illustrates the percentage of individuals who would have a differential classification by one glycemic measure as compared to the others (i.e. normal by fasting glucose, but impaired by OGTT etc.). When classifying individuals with HbA1c, $21.6 \%$ of individuals with impaired levels of $2 \mathrm{hPG}$ and $6.9 \%$ of individuals with type 2 diabetic levels of $2 \mathrm{hPG}$ would be misclassified as having normal glycemic levels. Similarly, FPG would misclassify $21.7 \%$ of individuals with impaired levels of $2 \mathrm{hPG}$ and $7.5 \%$ of individuals with type 2 diabetic levels of $2 \mathrm{hPG}$ as having normal glycemic levels. In other words, the positive predictive values of FPG and HbA1c were $35.1 \%$ and $39.7 \%$, respectively. The negative predictive values of FPG and $\mathrm{HbA} 1 \mathrm{c}$ was similar $(99.1 \%$ and $99.2 \%$, respectively) with respect to correctly classifying individuals who do not have type 2 diabetes.

The HR for all-cause and CVD mortality per SD increase of FPG, 2hPG and HbA1c were calculated. After adjusting for covariates, $2 \mathrm{hPG}$ had the highest HR for both all-cause (HR=1.22 [1.11-1.34]) and CVD

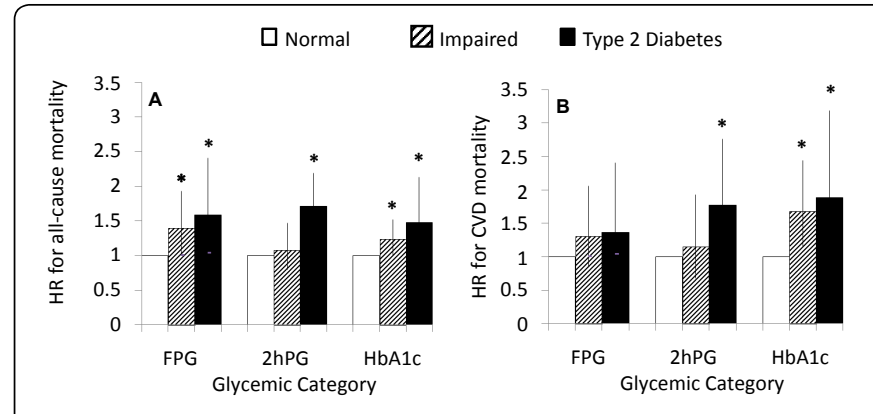

FPG (<5.6, 5.6-6.9 and $\geq 7.0 \mathrm{~m} \mathrm{~mol} / \mathrm{l}) ; 2 \mathrm{hPG}(<7.8,7.8-11.0,>11.0 \mathrm{~m} \mathrm{~mol} / \mathrm{l})$; HbA1c (<5.7, 5.7-6.4, $\geq 6.5 \%)$

Models are adjusted for age, sex, income, ethnicity, smoking status, alcohol intake, being physically active and high dietary fat intake ${ }^{*}=p<0.05$

Figure 2: Hazards ratios (95\% confidence intervals) for all-cause mortality (A) and CVD mortality (B) within each glycemic category of FPG, $2 \mathrm{hPG}$ and $\mathrm{HbA} 1 \mathrm{c}$ as compared to normal (referent).

$(\mathrm{HR}=1.23[1.04-1.46])$ mortality. HbA1c was only associated with an increased risk for CVD mortality $(\mathrm{HR}=1.19$ [1.05-1.35], but not all-cause mortality $(\mathrm{HR}=1.11$ [0.99-1.25]. FPG was not significantly associated with either all-cause $(\mathrm{HR}=1.11$ [1.00-1.23] or CVD $(\mathrm{HR}=1.12[0.95-1.31])$ mortality.

The HR for categories of FPG, 2hPG and HbA1c are presented in Figure 2. After adjustment for covariates, impaired and type 2 diabetic levels of all three glycemic measures were found to be significantly associated with a higher risk for all-cause mortality, with the exception of impaired levels of 2hPG. For CVD mortality, impaired and type 2 diabetic levels of HbA1c were found to be a significant predictor of mortality risk. However, only type 2 diabetic levels of 2hPG and not impaired levels were significantly associated with CVD mortality. FPG was not a significant predictor of CVD mortality risk.

\section{Discussion}

This study demonstrates that when using clinically relevant cutoffs for glycemia, $\mathrm{HbA} 1 \mathrm{c}$ is a more prognostic measure than $2 \mathrm{hPG}$ for both all-cause and CVD mortality risk. This finding is in contrast to previous work examining the association between glycemic measures as continuous variables and mortality risk. These findings support the use of $\mathrm{HbA} 1 \mathrm{c}$ in the clinical setting as an alternative to the OGTT for diagnosing diabetes. 
To date, there are only a small number of studies that have compared the ability of FPG, $2 \mathrm{hPG}$ and $\mathrm{HbA} 1 \mathrm{c}$ to predict all-cause and CVD mortality [12-17]. In these studies, the glycemic measures were examined as continuous variables, or categorized using distributional cutoffs. One study to date has examined the glycemic measures using relevant clinical cut-offs; however, the current study was the first to examine the mortality risk associated with each individual measure in a population representative sample. When each glycemic measure was analyzed as a continuous variable, we and others $[13,14]$ demonstrate that $2 \mathrm{hPG}$ was the measure most strongly associated with both allcause and CVD mortality risk. Similarly, a recent prospective cohort study of over 10,000 men and women demonstrate that $2 \mathrm{hPG}$ and FPG, rather than HbA1c was an independent predictor of both allcause and CVD mortality [12]. It is important to note however, that the clinical utility of this approach is limited, as in clinical practice, patients are generally diagnosed as having normal, impaired, or type 2 diabetic glycemic levels according to clinical cutoffs. Thus, it is important to understand the prognostic ability each cut-off has with respect to mortality.

In contrast to the results examining each glycemic measure as a continuous variable, $\mathrm{HbA} 1 \mathrm{c}$ appears as the measure that provides the most prognostic information for mortality risk when each measure is categorized using clinical cutoffs. We observed that unlike $2 \mathrm{hPG}$ or FPG, impaired and type 2 diabetic levels of HbA1c showed stepwise increases in all-cause and CVD mortality risk. These results are in contrast to a study conducted by de Vegt et al. [13], which also used clinically relevant cutoffs to categorize the three glycemic measures in older adults. After adjustments for all covariates their study found that only the type 2 diabetic category of $2 \mathrm{hPG}$ was significantly associated with all-cause mortality and no category of $2 \mathrm{hPG}$, FPG or HbA1c was associated with CVD mortality. As their study was conducted in a smaller sample of older men and women and used metabolic covariates as opposed to lifestyle covariates, it is possible that the discordant results may be attributable to the difference in the population or methods used between studies. Our results however, are in line with the growing literature that supports the use of HbA1c in the clinical setting $[4,22]$ and suggests that even modest elevations in HbA1c have cardiovascular consequences and thus should be treated at earlier prognostic stages than by $2 \mathrm{hPG}$ or FPG [23].

OGTT is established as the gold standard for diagnosing diabetes and has been demonstrated to be the better predictor of incident type 2 diabetes (as diagnosed by OGTT) as compared to HbA1c [21]. This introduces a clear bias, as the best predictor of any variable would be the variable itself. Further, these measures are inherently different in what they represent [5]. OGTT reflects the inability to properly manage blood glucose levels after a standardized glucose blood challenge, whereas HbA1c is the average glucose over the last three months. Thus theoretically, one could have a high HbA1c due to glucose intolerance, or due to a high glucose diet in the presence of normal glucose tolerance. Accordingly, one would expect differences in who may be classified as impaired or having type 2 diabetes by each measure. In the clinical setting, a diagnosis of diabetes is usually based on FPG or $2 \mathrm{hPG}$ levels. If HbA1c was used as the sole diagnostic measure, $32.7 \%$ of individuals with impaired or type 2 diabetic levels of FPG or $2 \mathrm{hPG}$ would be misclassified as having normal glycemic levels. Yet, it is important to note that the negative predictive value of $\mathrm{HbA} 1 \mathrm{c}$ is $99.2 \%$, therefore this measure correctly identifies essentially all individuals who do not have type 2 diabetes. It could also be argued from a mechanistic standpoint, that the reason why impaired glucose tolerance is detrimental for health is due to the elevated blood glucose [3]. Consequently, one may expect that $\mathrm{HbA} 1 \mathrm{c}$ better reflects the risk of long term micro and macro vascular complications. Given the ease of acquisition and the cost of overall assessment (i.e. time, analysis) [2], adoption of HbA1c also has practical advantages over $2 \mathrm{hPG}$ and its incorporation into clinical assessment should continue to be considered.

The strengths and limitations of this study warrant mention. This study was conducted in a large, ethnically diverse sample representative of the general U.S. population. However, baseline data for this study was collected 16 to 22 years ago. Since then, significant changes in the treatment of type 2 diabetes have occurred, particularly in the area of drug therapy [24]. Therefore, it is possible that the advances in medication that have occurred over the past 20 years may have influenced the results of this study. It should also be mentioned that our analysis did not adjust for other potential confounders including prevalent diseases and other co morbidities such as high blood pressure and hyperlipidemia. Adjusting for these variables in the analyses may lessen the association found between the measures of glycemia and mortality risk and may be inappropriate as they may fall in the causal pathway of why glycemia is associated with mortality risk. Further, that all analyses were adjusted for the same covariates does not influence the relative importance of each glycemic measure to mortality risk. Lastly, previous analyses from NHANES have reported ethnic differences in the risk of type 2 diabetes [25]. Although ethnicity was adjusted for in this analysis, due to sample size restrictions, we did not explore the potential ethnic differences associated with glycemia and mortality.

In conclusion, our results demonstrate that when using clinically relevant cutoffs for glycemia, HbA1c is the more prognostic measure for all-cause and CVD mortality. Therefore, given the greater ease and lower cost of measurement, HbA1c should be considered a useful and beneficial diagnostic and prognostic alternative screening tool in the clinical setting.

\section{Acknowledgements}

Conflicts of Interest: There are no conflicts of interest to disclose. The Centers for Disease Control and Prevention (CDC) funded and conducted the NHANES III study with mortality follow-up and made the data publicly available.

Author Contributions: JIR, CIA, MCR and JLK: Study concept and design, analysis and interpretation of the data and preparation of manuscript.

Sponsors Role: The CDC had no role in this study. The authors had full access to all of the data in the study and take responsibility for the integrity of the data and the accuracy of the data analysis.

\section{References}

1. Saudek CD, Herman WH, Sacks DB, Bergenstal RM, Edelman D, et al. (2008) A new look at screening and diagnosing diabetes mellitus. J Clin Endocrinol Metab 93: 2447-2453.

2. Bennett CM, Guo M, Dharmage SC (2007) $\mathrm{HbA}(1 \mathrm{c})$ as a screening tool for detection of Type 2 diabetes: a systematic review. Diabet Med 24: 333-343.

3. Unwin N, Shaw J, Zimmet P, Alberti KG (2002) Impaired glucose tolerance and impaired fasting glycaemia: the current status on definition and intervention. Diabet Med 19: 708-723.

4. Selvin E, Crainiceanu CM, Brancati FL, Coresh J (2007) Short-term variability in measures of glycemia and implications for the classification of diabetes. Arch Intern Med 167: 1545-1551.

5. Goldstein DE, Little RR, Lorenz RA (2004) Tests of glycemia in diabetes Diabetes Care 27: 1761-1773.

6. Suleiman M, Hammerman $H$, Boulos M, Kapeliovich MR, Suleiman A, et al (2005) Fasting glucose is an important independent risk factor for 30-day mortality in patients with acute myocardial infarction: a prospective study. Circulation 111: 754-760. 
Citation: Reddigan JI, Ardern Cl, Riddell MC, Kuk JL (2010) Differences in the Association between Clinically Relevant Classifications of Glycemia Measures and All-Cause and Cardiovascular Disease Mortality Risk. J Diabetes Metab 1:106. doi:10.4172/2155-6156.1000106

7. Barr EL, Zimmet PZ, Welborn TA, Jolley D, Magliano DJ, et al. (2007) Risk of cardiovascular and all-cause mortality in individuals with diabetes mellitus, impaired fasting glucose and impaired glucose tolerance: the Australian Diabetes, Obesity and Lifestyle Study (AusDiab). Circulation 116: 151-157.

8. Metter EJ, Windham BG, Maggio M, Simonsick EM, Ling SM, et al. (2008) Glucose and insulin measurements from the oral glucose tolerance test and mortality prediction. Diabetes Care 31: 1026-1030.

9. Glucose tolerance and mortality: comparison of WHO and American Diabetes Association diagnostic criteria. The DECODE study group. European Diabetes Epidemiology Group. Diabetes Epidemiology: Collaborative analysis Of Diagnostic criteria in Europe. (1999) Lancet 354: 617-621.

10. Khaw KT, Wareham N, Luben R, Bingham S, Oakes S, et al. (2001) Glycated haemoglobin, diabetes and mortality in men in Norfolk cohort of european prospective investigation of cancer and nutrition (EPIC-Norfolk). BMJ 322: 1518.

11. Khaw KT, Wareham N, Bingham S, Luben R, Welch A, et al. (2004) Association of hemoglobin A1c with cardiovascular disease and mortality in adults: the European prospective investigation into cancer in Norfolk. Ann Intern Med 141: 413-420.

12. Barr EL, Boyko EJ, Zimmet PZ, Wolfe R, Tonkin AM, Shaw JE (2009) Continuous relationships between non-diabetic hyperglycaemia and both cardiovascular disease and all-cause mortality: the Australian Diabetes, Obesity and Lifestyle (AusDiab) study. Diabetologia 52: 415-424.

13. de Vegt F, Dekker JM, Ruhe HG, Stehouwer CD, Nijpels G et al. (1999) Hyperglycaemia is associated with all-cause and cardiovascular mortality in the Hoorn population: the Hoorn Study. Diabetologia 42: 926-931.

14. Qiao Q, Dekker JM, de Vegt F, Nijpels G, Nissinen A, et al. (2004) Two prospective studies found that elevated 2-hr glucose predicted male mortality independent of fasting glucose and HbA1c. J Clin Epidemiol 57: 590-596.

15. Meigs JB, Nathan DM, D'Agostino RB S, Wilson PW, Framingham Offspring Study (2002) Fasting and postchallenge glycemia and cardiovascular disease risk: the Framingham Offspring Study. Diabetes Care 25: 1845-1850.
16. Park S, Barrett-Connor E, Wingard DL, Shan J, Edelstein S (1996) GHb is a better predictor of cardiovascular disease than fasting or postchallenge plasma glucose in women without diabetes. The Rancho Bernardo Study. Diabetes Care 19: 450-456.

17. Cohen BE, Barrett-Connor E, Wassel CL, Kanaya AM (2009) Association of glucose measures with total and coronary heart disease mortality: does the effect change with time? The Rancho Bernardo Study. Diabetes Res Clin Pract 86: $67-73$

18. Skeie S, Thue G, Sandberg S (2000) Use and interpretation of HbA1c testing in general practice. Implications for quality of care. Scand J Clin Lab Invest 60: 349-356.

19. Sarwar N, Aspelund T, Eiriksdottir G, Gobin R, Seshasai SR, et al. (2010) Markers of dysglycaemia and risk of coronary heart disease in people without diabetes: Reykjavik prospective study and systematic review. PLoS Med 7: e1000278.

20. NHANES - Analytical Guidelines Available from:http://www.cdc.gov/nchs/ nhanes/nhanes2003-2004/analytical_guidelines.htm, accessed 7/8/2010 2010.

21. American Diabetes Association (2010) Diagnosis and classification of diabetes mellitus. Diabetes Care 33 : S62-69.

22. Adams RJ, Appleton SL, Hill CL, Wilson DH, Taylor AW, et al. (2009) Independent association of $\mathrm{HbA}(1 \mathrm{c})$ and incident cardiovascular disease in people without diabetes. Obesity (Silver Spring) 17: 559-563.

23. Whiteley L, Padmanabhan S, Hole D, Isles C (2005) Should diabetes be considered a coronary heart disease risk equivalent?: results from 25 years of follow-up in the Renfrew and Paisley survey. Diabetes Care 28: 1588-1593.

24. Stolar MW, Hoogwerf BJ, Gorshow SM, Boyle PJ, Wales DO (2008) Managing type 2 diabetes: going beyond glycemic control. J Manag Care Pharm 14: s219.

25. Harris MI, Flegal KM, Cowie CC, Eberhardt MS, Goldstein DE, et al. (1998) Prevalence of diabetes, impaired fasting glucose and impaired glucose tolerance in U.S. adults. The Third National Health and Nutrition Examination Survey, 1988-1994. Diabetes Care 21: 518-524. 